C-A/AP/\#144

August 2005

\title{
Results for the intrabeam scattering growth Rates for a bi-gaussian distribution
}

\author{
George Parzen
}

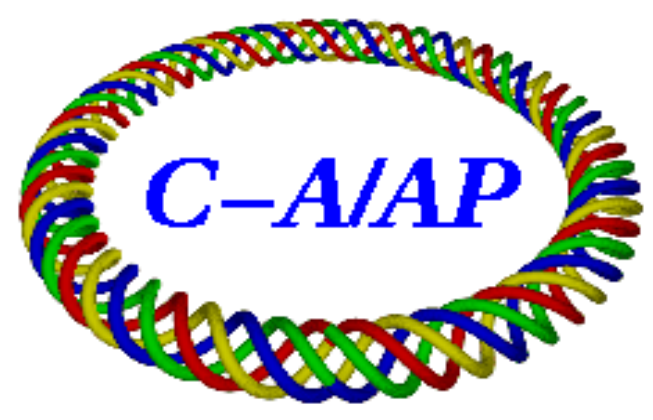

Collider-Accelerator Department Brookhaven National Laboratory Upton, NY 11973 


\title{
Results for the intrabeam scattering growth rates for a bi-gaussian distribution.
}

\author{
George Parzen \\ March 2004 \\ BNL Report C-A/AP No.144
}

\begin{abstract}
This note lists results for the intrabeam scattering growth rates for a bi-gaussian distribution. The derivation of these results will be given in a future note.
\end{abstract}

\section{Introduction}

This note finds results for the intrabeam scattering growth rates for a bigaussian distribution.

The bi-gaussian distribution is interesting for studying the possibility of using electron cooling in RHIC. Studies done using the SIMCOOL program [1] indicate that in the presence of electron cooling, the beam distribution changes so that it developes a strong core and a long tail which is not described well by a gaussian, but may be better described by a bi-gaussian. Being able to compute the effects of intrabeam scattering for a bi-gaussian distribution would be useful in computing the effects of electron cooling, which depend critically on the details of the intrabeam scattering.

\section{Gaussian distribution}

Before defining the bi-gaussian distribution, the gaussian distribution will be reviewed. 
$N f(x, p)$ gives the number of particles in $d^{3} x d^{3} p$, where $\mathrm{N}$ is the number of particles in a bunch. For a gaussian distribution, $f(x, p)$ ls given by

$$
\begin{aligned}
& f(x, p)=\frac{1}{\Gamma} \exp [-S(x, p)] \\
S & =S_{x}+S_{y}+S_{s} \\
S_{x} & =\frac{1}{\overline{\epsilon_{x}}} \epsilon_{x}\left(x_{\beta}, p_{x \beta} / p_{0}\right) \\
x_{\beta} & =x-D\left(p-p_{0}\right) / p_{0} \\
p_{x \beta} / p_{0} & =p_{x} / p_{0}-D^{\prime}\left(p-p_{0}\right) / p_{0} \\
\epsilon_{x}\left(x, x^{\prime}\right) & =\gamma_{x} x^{2}+2 \alpha_{x} x x^{\prime}+\beta_{x} x^{\prime 2} \\
S_{y} & =\frac{1}{\overline{\epsilon_{y}}} \epsilon_{y}\left(y, p_{y} / p_{0}\right) \\
\epsilon_{y}\left(y, y^{\prime}\right) & =\gamma_{y} y^{2}+2 \alpha_{y} y y^{\prime}+\beta_{y} y^{2} \\
S_{s} & =\frac{1}{2 \sigma_{s}^{2}}\left(s-s_{c}\right)^{2}+\frac{1}{2 \sigma_{p}^{2}}\left(\left(p-p_{0}\right) / p_{0}\right)^{2} \\
S_{s} & =\frac{1}{\bar{\epsilon}_{s}}\left(\frac{1}{\beta_{s}}\left(s-s_{c}\right)^{2}+\beta_{s}\left(\left(p-p_{0}\right) / p_{0}\right)^{2}\right. \\
\beta_{s} & =\sigma_{s} / \sigma_{p} \\
\bar{\epsilon}_{s} & =2 \sigma_{s} \sigma_{p} \\
S_{s} & =\frac{1}{\bar{\epsilon}_{s}} \epsilon_{s}\left(s-s_{c},\left(p-p_{0}\right) / p_{0}\right) \\
\Gamma & =\int d^{3} x d^{3} p \exp [-S(x, p)] \\
\Gamma & =\pi^{3} \overline{\epsilon_{x}} \bar{\epsilon}_{y} \bar{\epsilon}_{s} p_{0}^{3} \\
\overline{\epsilon_{i}} & =<\epsilon_{i}(x, p)>i=x, y, s
\end{aligned}
$$

$D$ is the horizontal dispersion. $D^{\prime}=d D / d s$. $<>$ indicates an average over all the particles in a bunch.

\section{Growth rates for a Gaussian distribution}

In the following,the growth rates are given in the Rest Coordinate System, which is the coordinate system moving along with the bunch. Growth rates 
are given for $\left\langle p_{i} p_{j}\right\rangle$. From these one can compute the growth rates for $<\epsilon_{i}>$ using the relations given at the end of this note.

$$
\begin{aligned}
\frac{1}{p_{0}^{2}} \frac{d}{d t}<p_{i} p_{j}> & =\frac{N}{\Gamma} \int d^{3} \Delta \exp [-R] C_{i j} \\
C_{i j} & =\frac{2 \pi}{p_{0}^{2}}\left(r_{0} / 2 \bar{\beta}^{2}\right)^{2}\left(|\Delta|^{2} \delta_{i j}-3 \Delta_{i} \Delta_{j}\right) 2 \bar{\beta} c \ln \left[1+\left(2 \bar{\beta}^{2} b_{m a x} / r_{0}\right)^{2}\right] \\
\bar{\beta} & =\beta_{0} \gamma_{0}\left|\Delta / p_{0}\right| \\
r_{0} & =Z^{2} e^{2} / M c^{2} \\
R & =R_{x}+R_{y}+R_{s} \\
R_{x} & =\frac{2}{\beta_{x} \overline{\epsilon_{x}}}\left[\gamma^{2} D^{2} \Delta_{s}^{2}+\left(\beta_{x} \Delta_{x}-\gamma \tilde{D} \Delta_{s}\right)^{2}\right] / p_{0}^{2} \\
\tilde{D} & =\beta_{x} D^{\prime}+\alpha_{x} D \\
R_{y} & =\frac{2}{\beta_{y} \bar{\epsilon}_{y}} \beta_{y}^{2} \Delta_{y}^{2} / p_{0}^{2} \\
R_{s} & =\frac{2}{\beta_{s} \bar{\epsilon}_{s}} \beta_{s}^{2} \gamma^{2} \Delta_{s}^{2} / p_{0}^{2}
\end{aligned}
$$

The integral over $d^{3} \Delta$ is an integral over all possible values of the relative momemtum for any two particles in a bunch. $\beta_{0}, \gamma_{0}$ are the beta and gamma corresponding to $p_{0}$, the central momemtum of the bunch in the Laboratory Coordinate System. $\gamma=\gamma_{0}$

The above 3-dimensional integral can be reduced to a 2-dimensional integral by integrating over $|\Delta|$ and using $d^{3} \Delta=|\Delta|^{2} d|\Delta| \sin \theta d \theta d \phi$.

$$
\begin{aligned}
\frac{1}{p_{0}^{2}} \frac{d}{d t}<p_{i} p_{j}>= & \frac{N}{\Gamma} 2 \pi p_{0}^{3}\left(\frac{r_{0}}{2 \gamma_{0}^{2} \beta_{0}^{2}}\right)^{2} 2 \beta_{0} \gamma_{0} c \int \sin \theta d \theta d \phi\left(\delta_{i j}-3 g_{i} g_{j}\right) \\
& \frac{1}{F} \ln \left[\frac{\hat{C}}{F}\right] \\
g_{3}= & \cos \theta=g_{s} \\
g_{1}= & \sin \theta \cos \phi=g_{x} \\
g_{2}= & \sin \theta \sin \phi=g_{y} \\
\hat{C}= & 2 \gamma_{0}^{2} \beta_{0}^{2} b_{\max } / r_{0} \\
F & =R /\left(|\Delta| / p_{0}\right)^{2}
\end{aligned}
$$




$$
\begin{aligned}
F & =F_{x}+F_{y}+F_{s} \\
F_{x} & =\frac{2}{\beta_{x} \overline{\epsilon_{x}}}\left[\gamma^{2} D^{2} g_{s}^{2}+\left(\beta_{x} g_{x}-\gamma \tilde{D} g_{s}\right)^{2}\right] \\
F_{y} & =\frac{2}{\beta_{y} \overline{\epsilon_{y}}} \beta_{y}^{2} g_{y}^{2} \\
F_{s} & =\frac{2}{\beta_{s} \overline{\epsilon_{s}}} \beta_{s}^{2} \gamma^{2} g_{s}^{2}
\end{aligned}
$$

\section{Bi-Gaussian distribution}

The bi-gaussian distribution will be assumed to have the form given by the following.

$N f(x, p)$ gives the number of particles in $d^{3} x d^{3} p$, where $\mathrm{N}$ is the number of particles in a bunch. For a bi-gaussian distribution, $f(x, p)$ ls given by

$$
f(x, p)=\frac{N_{a}}{N} \frac{1}{\Gamma_{a}} \exp \left[-S_{a}(x, p)\right]+\frac{N_{b}}{N} \frac{1}{\Gamma_{b}} \exp \left[-S_{b}(x, p)\right.
$$

In the first gaussian,to find $\Gamma_{a}, S_{a}$ then in the expressions for $\Gamma, S$, given above for the gaussian distribution, replace $\overline{\epsilon_{x}}, \overline{\epsilon_{y}}, \overline{\epsilon_{s}}$ by $\epsilon_{x a}^{-}, \epsilon_{y a}^{-}, \epsilon_{s a}^{-}$. In the second gaussian, in the expressions for $\Gamma, S$, replace $\overline{\epsilon_{x}}, \overline{\epsilon_{y}}, \overline{\epsilon_{s}}$ by $\bar{\epsilon}_{x b}^{-}, \overline{\epsilon_{y b}}, \overline{\epsilon_{s b}}$. In addition. $N_{a}+N_{b}=N$. This bi-gaussian has 7 parameters instead of the three parameters of a gaussian.

\section{Growth rates for a Bi- Gaussian distribution}

In the following,the growth rates are given in the Rest Coordinate System, which is the coordinate system moving along with the bunch. Growth rates are given for $<p_{i} p_{j}>$. From these one can compute the growth rates for $<\epsilon_{i}>$ using the relations given at the end of this note.

$$
\begin{aligned}
\frac{1}{p_{0}^{2}} \frac{d}{d t}<p_{i} p_{j}>= & N \int d^{3} \Delta C_{i j}\left[\left(\frac{N_{a}}{N}\right)^{2} \frac{\exp \left(-R_{a}\right)}{\Gamma_{a}}+\left(\frac{N_{b}}{N}\right)^{2} \frac{\exp \left(-R_{b}\right)}{\Gamma_{b}}\right. \\
& \left.+2 \frac{N_{a} N_{b}}{N^{2}} \frac{\Gamma_{c}}{\Gamma_{a} \Gamma_{b}} \exp (-T)\right] \\
C_{i j}= & \frac{2 \pi}{p_{0}^{2}}\left(r_{0} / 2 \bar{\beta}^{2}\right)^{2}\left(|\Delta|^{2} \delta_{i j}-3 \Delta_{i} \Delta_{j}\right) 2 \bar{\beta} c \ln \left[1+\left(2 \bar{\beta}^{2} b_{\max } / r_{0}\right)^{2}\right]
\end{aligned}
$$




$$
\begin{aligned}
& \bar{\beta}=\beta_{0} \gamma_{0}\left|\Delta / p_{0}\right| \\
& r_{0}=Z^{2} e^{2} / M c^{2} \\
& \frac{1}{\overline{\epsilon_{i c}}}=\frac{1}{2}\left(\frac{1}{\overline{\epsilon_{i a}}}+\frac{1}{\overline{\epsilon_{i b}}}\right) \quad i=x, y, s \\
& \frac{1}{\bar{\epsilon}_{i d}}=\frac{1}{2}\left(\frac{1}{\bar{\epsilon}_{i a}}-\frac{1}{\bar{\epsilon}_{i b}}\right) \\
& r_{0}=Z^{2} e^{2} / M c^{2} \\
& \Gamma_{a}=\pi^{3} \epsilon_{s a}^{-} \epsilon_{x a}^{-} \epsilon_{y a}^{-} p_{0}^{3} \\
& R_{a}=R_{x a}+R_{y a}+R_{s a} \\
& R_{x a}=\frac{2}{\beta_{x} \overline{\epsilon_{x}}}\left[\gamma^{2} D^{2} \Delta_{s}^{2}+\left(\beta_{x} \Delta_{x}-\gamma \tilde{D} \Delta_{s}\right)^{2}\right] / p_{0}^{2} \\
& \tilde{D}=\beta_{x} D^{\prime}+\alpha_{x} D \\
& R_{y a}=\frac{2}{\beta_{y} \bar{\epsilon}_{y}} \beta_{y}^{2} \Delta_{y}^{2} / p_{0}^{2} \\
& R_{s a}=\frac{2}{\beta_{s} \overline{\epsilon_{s}}} \beta_{s}^{2} \gamma^{2} \Delta_{s}^{2} / p_{0}^{2} \\
& T=T_{x}+T_{y}+T_{s} \\
& T_{x}=R_{x c}-R_{x d} \\
& T_{y}=R_{y c}-R_{y d} \\
& T_{s}=R_{s c}-R_{s d} \\
& R_{x d}=2\left\{\left[-\gamma D \bar{\Delta}_{s}\right]^{2}\right. \\
& \left.+\left[\left(\beta_{x} \bar{\Delta}_{x}-\gamma \bar{D} \bar{\Delta}_{s}\right)\right]^{2}\right\} \\
& /\left(\beta_{x} \bar{\epsilon}_{x d}^{2} / \bar{\epsilon}_{x c}\right) \\
& R_{y d}=\frac{2 \beta_{y}}{\bar{\epsilon}_{y d}^{2} / \bar{\epsilon}_{y c}} \bar{\Delta}_{y}^{2} \\
& R_{s d}=\frac{2 \beta_{s}}{\bar{\epsilon}_{s d}^{2} / \bar{\epsilon}_{s c}} \bar{\Delta}_{s}^{2} \\
& \bar{\Delta}_{i}=\Delta_{i} / p_{0} \quad i=x, y, s
\end{aligned}
$$

$R_{a}, R_{b}, R_{c}$ are each the same as $R_{a}$ given above except that $\overline{\epsilon_{i a}}$ are replaced 
by $\overline{\epsilon_{i a}}, \overline{\epsilon_{i b}}, \overline{\epsilon_{i c}}$ respectively.

The above 3-dimensional integral can be reduced to a 2-dimensional integral by integrating over $|\Delta|$.

$$
\begin{aligned}
\frac{1}{p_{0}^{2}} \frac{d}{d t}<p_{i} p_{j}>= & 2 \pi p_{0}^{3}\left(\frac{r_{0}}{2 \gamma_{0}^{2} \beta_{0}^{2}}\right)^{2} 2 \beta_{0} \gamma_{0} c \int \sin \theta d \theta d \phi\left(\delta_{i j}-3 g_{i} g_{j}\right) \\
& N\left[\left(\frac{N_{a}}{N}\right)^{2} \frac{1}{\Gamma_{a} F_{a}} \ln \left[\frac{\hat{C}}{F_{a}}\right]+\left(\frac{N_{b}}{N}\right)^{2} \frac{1}{\Gamma_{b} F_{b}} \ln \left[\frac{\hat{C}}{F_{b}}\right]\right. \\
& \left.+2 \frac{N_{a} N_{b}}{N^{2}} \frac{\Gamma_{c}}{\Gamma_{a} \Gamma_{b}} \frac{1}{G} \ln \left[\frac{\hat{C}}{G}\right]\right] \\
g_{3}= & \cos \theta=g_{s} \\
g_{1}= & \sin \theta \cos \phi=g_{x} \\
g_{2}= & \sin \theta \sin \phi=g_{y} \\
\hat{C}= & 2 \gamma_{0}^{2} \beta_{0}^{2} b_{\max } / r_{0} \\
F_{i}= & R_{i} /\left(|\Delta| / p_{0}\right)^{2} \quad i=a, b, c \\
G= & T /\left(|\Delta| / p_{0}\right)^{2}
\end{aligned}
$$

$F_{a}, F_{b}, F_{c}$ are each the same $\mathrm{F}$ that was defined for the Gaussian distribution except that the $\overline{\epsilon_{i}}$ are replaced by $\overline{\epsilon_{i a}}, \overline{\epsilon_{i b}}, \overline{\epsilon_{i c}}$ respectively.

The above results for the growth rates for a bi-gaussian distribution are expressed as an integral which contains 3 terms, each of which is similar to the one term in the results for the gaussian distribution. These three terms may be given a simple interpertation. The first term represents the contribution to the growth rates due to the scattering of the $N_{a}$ particles of the first gaussian from themselves, the seond term the contribution due to the scattering of the $N_{b}$ particles of the second gaussian from themselves, and the third term the contribution due to the scattering of the $N_{a}$ particles of the first gaussian from the $N_{b}$ partcles of the second gaussian. 


\section{Emittance growth rates}

One can compute growth rates for the average emittances, $\left\langle\epsilon_{i}\right\rangle$ in the Laboratory Coordinate System, from the growth rates for $\left\langle p_{i} p_{j}\right\rangle$ in the Rest Coordinate System.In the following, $d t$ is the time interval in the Laboratory System and $d \tilde{t}$ is the time interval in the Rest System. $d t=\gamma d \tilde{t}$

$$
\begin{aligned}
\frac{d}{d t} \epsilon_{x} & =\frac{\beta_{x}}{\gamma} \frac{d}{d \tilde{t}}<p_{x}^{2} / p_{0}^{2}>+\frac{D^{2}+\tilde{D}^{2}}{\beta_{x}} \gamma \frac{d}{d \tilde{t}}<p_{s}^{2} / p_{0}^{2}>-2 \tilde{D} \frac{d}{d \tilde{t}}<p_{x} p_{s} / p_{0}^{2}> \\
\frac{d}{d t} \epsilon_{y} & =\frac{\beta_{y}}{\gamma} \frac{d}{d \tilde{t}}<p_{y}^{2} / p_{0}^{2}> \\
\frac{d}{d t} \epsilon_{s} & =\beta_{s} \gamma \frac{d}{d \tilde{t}}<p_{s}^{2} / p_{0}^{2}>
\end{aligned}
$$

I thank I. Ben-Zvi for his comments and encouragement. The results given above were found using the results given in references $[2,3,4$,$] .The derivation$ of the results is given in Ref.[5]

\section{References}

1. V.Parhomchhuk and I. Ben-Zvi, BNL report C-A/AP/47, April 2001; A. Fedotov, Y. Eidelman (Private Communication 2004)

2. G.Parzen, BNL report C-A/AP/No.150 (2004)

3. A. Piwinski Proc. 9th Int. Conf. on High Energy Accelerators (1974) 405, M. Martini CERN PS/84-9 (1984), A. Piwinski Cern 87-03 (1987) 402, A. Piwinski CERN 92-01 (1992) 226

4. J.D. Bjorken and S.K. Mtingwa, Part. Accel.13 (1983) 115, K. Kubo and K. Oide Phys. Rev. S.T.A.B., 4, (2001) 124401

5. G. Parzen, BNL report C-A/AP/No.169 (2004) 